\title{
EMERGENCY MEDICAL SERVICES AND ROAD CONGESTION: DEADLY PATH TO HOSPITALS
}

\author{
Khalid Mahmood ${ }^{1}$ and Nazila Bano Khalid ${ }^{2}$
}

${ }^{1}$ Department of Radiology, Sindh Institute of Urology and Transplantation (SIUT), Karachi, Pakistan

${ }^{2}$ The Caravan of Life Pakistan Trust, The Recovery House (Psychiatric Rehabilitation Service), Karachi, Pakistan.

Correspondence: Khalid Mahmood, E-mail: Khalid.rad456@gmail.com, Cell: 0323-2001779

\begin{abstract}
Summary
The emergency medical services (EMS) are essential services and time is the crucial factor in saving lives and reducing morbidities. The spatial and temporal differences of EMS accessibility are utmost priority for the assessment, provision and efficient emergency service dispatch in densely populated urban communities of low to middle income countries. The EMS of mega populous cities like Karachi manifests the oblivious of governance to such essential and functional collaboration.

The impact of traffic congestion and gridlock on EMS accessibility is significant in areas where there unending development projects, broken road networks and traffic rules are ignored. No steps are taken to clear the congestion and repair the roads in front of hospitals. It calls for the collaboration of EMS and urban development and planning authorities, health care services, disaster management, and ministry of traffic and transport.
\end{abstract}

Keywords: Emergency medical-service (ems), urban canyons, traffic congestion, urban development, accessibility

Traffic congestion, poor road networks and unplanned urbanization are growing grave concerns for provision and accessibility of emergency medical care services in thickly populated cities of low and middle income countries (LMICs) like Karachi. The spatial and temporal differences of Emergency Medical services (EMS) accessibility are vital for the assessment, provision and utilization of emergency facilities and the efficiency of emergency service dispatch in the urban communities. This mega metropolis city of Pakistan depicts unplanned urbanization resulting in formation of urban canyons, impeding the accessibility to emergency medical care services

This was a cross-sectional exploratory study conducted from March-May 2017 to analyze the impact of traffic congestion and unplanned construction of buildings on the temporal and spatial accessibility to EMS in Karachithe economic and industrial hub of Pakistan. The target study area was Saddar Town- Karachi, the heart of all tertiary and disaster management healthcare services. The analysis was done by using the elevated observation and photographic impressions during peak hours of weekdays, in-depth-interviews with EMS transportation personal and traffic constables and maximum-car-methods.

The study revealed that the roads leading to major city hospitals including; Burns and ShaheedMuhtarma Benazir Bhutto (SMBB) Trauma Centre, Sindh Institute of Urology and Transplantation (SIUT), Civil Hospital(Tertiary Care Hospital), Sind Services Hospital(Secondary Care Hospital), Sobhraj and Lady Defren Hospitals(Hospitals for Women), are inaccessible due to road works and traffic congestion. And, there have been no steps to clear the congestion and repair the roads in front of hospitals. The road from Pakistan Chowk to Dow Medical College (DMC) is uneven and offers a bumpy ride to the patients brought to the hospital. Heavy traffic congestion due to overcrowding of vehicles around Dow Medical College area is the root cause of the problem.

The results revealed that: (1) During rush hours, the average and longest time between 12 hospitals and communities are $80 \%$ higher than using standard road speed (2) The accessibility of EMS to an emergency hospital is poor due to bumpy road network and bottleneck situations 1 . There is an inverse relationship between accessibility of EMS and areas where the emergency hospital number is large and the road network is dense. The impact of traffic congestion and gridlock on EMS accessibility is significant in areas where the road network are broken and traffic rules are ignored2 (3) with the increase of time impedance, accessibility was slowed. This trend is more obvious in off-rush hours

Time is a sensitive and crucial factor in emergency medical service (EMS). Traffic congestion and gridlocks 
are common problem in the central areas of mega populous cities. The megacities in low and middle income countries (LMICs) like Karachi are vulnerable to man-made disasters like bomb blasts and ever growing road traffic incidents. The spatial and temporal differences of EMS accessibility are vital for the assessment, provision and utilization of emergency facilities and the efficiency of emergency service dispatch in the urban communities

Traffic congestion, poor road networks and unplanned urbanization are growing grave concerns for provision and accessibility of emergency medical care services in thickly populated cities of low and middle income countries (LMICs) like Karachi. This mega metropolis city of Pakistan depicts unplanned urbanization resulting in formation of urban canyons, impeding the accessibility to emergency medical care services

\section{Public Health Implications}

The real-time traffic condition should be considered in development and provision of EMS to optimize EMS scheduling and improve the efficiency to ensure accessibility to non-affording consumers and victims of disaster (bomb blasts, road traffic accidents) or natural calamity. The limited accessibility and unavailability of EMS services for communities result in compromised quality of care and health outcomes as well as less than optimal utilization of services

\section{References}

1. CHEN Jianguo, ZHOU Suhong, LIU Lin, et al Estimating the effect of traffic congestion on accessibility to emergency medical care services: Take Guangzhou as an example [J] Progress In Geography, 2016, 35 (4): 431-439

2. Brabyn L, Skelly C. Modeling population access to New Zealand public hospitals. International Journal of Health Geographics. 2002;1:3. doi:10.1186/1476-072X-1-3 